\title{
The binomial ideal of the intersection axiom for conditional probabilities
}

\author{
Alex Fink
}

Received: 10 February 2009 / Accepted: 27 August 2010 / Published online: 17 September 2010

(C) The Author(s) 2010. This article is published with open access at Springerlink.com

\begin{abstract}
The binomial ideal associated with the intersection axiom of conditional probability is shown to be radical and is expressed as an intersection of toric prime ideals. This solves a problem in algebraic statistics posed by Cartwright and Engström.
\end{abstract}

Keywords Conditional independence · Intersection axiom

Conditional independence constraints are a family of natural constraints on probability distributions, describing situations in which two random variables are independently distributed given knowledge of a third. Statistical models built around considerations of conditional independence, in particular graphical models in which the constraints are encoded in a graph on the random variables, enjoy wide applicability in determining relationships among random variables in statistics and in dealing with uncertainty in artificial intelligence.

One can take a purely combinatorial perspective on the study of conditional independence, as does Studený [10], conceiving of it as a relation on triples of subsets of a set of observables which must satisfy certain axioms. A number of elementary implications among conditional independence statements are recognized as axioms. Among these are the semi-graphoid axioms, which are implications of conditional independence statements lacking further hypotheses, and hence are purely combinatorial statements. The intersection axiom is also often added to the collection, but unlike the semi-graphoid axioms it is not uniformly true; it is our subject here.

Formally, a conditional independence model $\mathcal{M}$ is a set of probability distributions characterized by satisfying several conditional independence constraints. We

\footnotetext{
A. Fink (ه)

Department of Mathematics, North Carolina State University, Raleigh, NC, USA

e-mail: arfink@ncsu.edu
} 
will work in the discrete setting, where a probability distribution $p$ is a multi-way table of probabilities, and we follow the notational conventions in [1].

Consider the discrete conditional independence model $\mathcal{M}$ given by

$$
\left\{X_{1} \Perp X_{2}\left|X_{3}, X_{1} \Perp X_{3}\right| X_{2}\right\}
$$

where $X_{i}$ is a random variable taking values in the set $\left[r_{i}\right]=\left\{1, \ldots, r_{i}\right\}$. Throughout we assume $r_{1} \geq 2$. Let $p_{i j k}$ be the unknown probability $P\left(X_{1}=i, X_{2}=j, X_{3}=k\right)$ in a distribution from the model $\mathcal{M}$. The set of distributions in the model $\mathcal{M}$ is the variety whose defining ideal $I_{\mathcal{M}} \subseteq S=\mathbb{C}\left[p_{i j k}\right]$ is

$$
\begin{aligned}
I_{\mathcal{M}}= & \left(p_{i j k} p_{i^{\prime} j^{\prime} k}-p_{i j^{\prime} k} p_{i^{\prime} j k}: i, i^{\prime} \in\left[r_{1}\right], j, j^{\prime} \in\left[r_{2}\right], k \in\left[r_{3}\right]\right) \\
& +\left(p_{i j k} p_{i^{\prime} j k^{\prime}}-p_{i j k^{\prime}} p_{i^{\prime} j k}: i, i^{\prime} \in\left[r_{1}\right], j \in\left[r_{2}\right], k, k^{\prime} \in\left[r_{3}\right]\right) .
\end{aligned}
$$

The intersection axiom is the implication whose premises are the statements of $\mathcal{M}$ and whose conclusion is $X_{1} \Perp\left(X_{2}, X_{3}\right)$. To be true, this implication requires the further hypothesis that the distribution $p$ is in the interior of the probability simplex, i.e. that no individual probability $p_{i j k}$ is zero. It is thus a natural question to ask what can be inferred about distributions $p$ which may lie on the boundary of the probability simplex. In algebraic terms, we are asking for the (set-theoretic) components of the variety $V\left(I_{\mathcal{M}}\right)$.

A problem posed by Dustin Cartwright and Alexander Engström appears in Sect. 6.6.3 of [1], giving a conjectural description of the associated primes of $I_{\mathcal{M}}$ in terms of certain subgraphs of a complete bipartite graph. Our main theorem resolves this conjecture in the positive, and gives stronger information, namely the primary decomposition of $I_{\mathcal{M}}$.

In the course of this project the author computed primary decompositions of $I_{\mathcal{M}}$ for various values of $r_{1}, r_{2}$, and $r_{3}$ with the computer algebra system Singular $[4,5]$. Thomas Kahle has recently written dedicated Macaulay2 code [3] for binomial primary decompositions [7], in which the same computations may be carried out.

A broad generalization of this paper's results to the class of binomial edge ideals of graphs has been obtained by Herzog, Hibi, Hreinsdóttir, Kahle, and Rauh [6]. The $r=2$ case of $I_{\mathcal{M}}$ is treated, with a different term order, in their Sect. 4.

Let $K_{p, q}$ be the complete bipartite graph with bipartitioned vertex set $[p] \amalg[q]$. Given a subgraph $G$ of $K_{r_{2}, r_{3}}$ with edge set $\operatorname{Edges}(G)$, the prime $P_{G}$ to which it corresponds is defined to be

$$
P_{G}=P_{G}^{(0)}+P_{G}^{(1)}
$$

where

$$
\begin{aligned}
& P_{G}^{(0)}=\left(p_{i j k}: i \in\left[r_{1}\right],(j, k) \notin \operatorname{Edges}(G)\right), \\
& P_{G}^{(1)}=\left(p_{i j k} p_{i^{\prime} j^{\prime} k^{\prime}}-p_{i j^{\prime} k^{\prime}} p_{i^{\prime} j k}: i, i^{\prime} \in\left[r_{1}\right] ;\right. \text { and } \\
&\left.j, j^{\prime} \in\left[r_{2}\right], k, k^{\prime} \in\left[r_{3}\right] \text { are in the same connected component of } G\right) .
\end{aligned}
$$


Note that $j$ need not be distinct from $j^{\prime}$, nor $k$ from $k^{\prime}$. We will also want to refer to the individual summands $P_{C}^{(1)}$ of $P_{G}^{(1)}$, where $P_{C}^{(1)}$ includes only the generators $\left\{p_{i j k}:(j, k) \in C\right\}$ arising from edges in the connected component $C$ of $G$. Then

$$
P_{G}=P_{G}^{(0)}+\sum_{C} P_{C}^{(1)},
$$

where $C$ runs over connected components of $G$.

We say that a subgraph $G$ of $K_{r_{2}, r_{3}}$ is admissible if $G$ has vertex set [ $\left.r_{2}\right] \amalg\left[r_{3}\right]$ and all connected components of $G$ are isomorphic to some complete bipartite graph $K_{p, q}$ with $p, q \geq 1$.

Let $\prec_{\text {dp }}$ be the revlex term order on $S$ over the lexicographic variable order on subscripts, with earlier subscripts more significant. Thus under $\prec_{\mathrm{dp}}$, we have $p_{111} \prec_{\mathrm{dp}} p_{112} \prec_{\mathrm{dp}} p_{211}$.

Theorem 1 The primary decomposition

$$
I_{\mathcal{M}}=\bigcap_{G} P_{G}
$$

holds and is an irredundant decomposition, where the union is over admissible graphs $G$ on $\left[r_{2}\right] \amalg\left[r_{3}\right]$. We also have

$$
\operatorname{in}_{\prec \mathrm{dp}} I_{\mathcal{M}}=\bigcap_{G} \operatorname{in}_{\prec_{\mathrm{dp}}} P_{G}
$$

Each in $_{\prec_{\mathrm{dp}}} P_{G}$ is squarefree, so in $\prec_{\mathrm{dp}} I_{\mathcal{M}}$ and hence $I_{\mathcal{M}}$ are radical ideals.

In particular, the value of $r_{1}$ is irrelevant to the combinatorial nature of the primary decomposition.

Corollary 2 (Conjecture, Cartwright-Engström) The set of minimal primes of the ideal $I_{\mathcal{M}}$ is

$$
\left\{P_{G}: G \text { an admissible graph on }\left[r_{2}\right] \amalg\left[r_{3}\right]\right\} \text {. }
$$

Remark 3 This corollary amounts to the set-theoretic identity

$$
V\left(I_{\mathcal{M}}\right)=\bigcup_{G \text { admissible }} V\left(P_{G}\right)
$$

Points $\left(p_{i j k}\right)$ on the variety $V\left(P_{G}\right)$ are characterized by the conditions that $p_{i j k}=0$ for $(j, k) \notin \operatorname{Edges}(G)$, and that for any two edges $(j, k)$ and $\left(j^{\prime}, k^{\prime}\right)$ in the same connected component of $G$, the vectors $(p \cdot j k)$ and $\left(p \cdot j^{\prime} k^{\prime}\right)$ in $\mathbb{C}^{r_{1}}$ are proportional.

The core ideas of a proof of Corollary 2 are present in [1, Sect. 6.6.4]. That discussion focuses on the prime $P_{K_{2}, r_{3}}$, corresponding to the locus where the conclusion of the intersection axiom is valid, but it extends without great difficulty to any $P_{G}$. 
It is noted in $[1, \S 6.6]$ that the number $\eta(p, q)$ of admissible graphs $G$ on $[p] \amalg[q]$ is given by the generating function

$$
\exp \left(\left(e^{x}-1\right)\left(e^{y}-1\right)\right)=\sum_{p, q \geq 0} \eta(p, q) \frac{x^{p} y^{q}}{p ! q !},
$$

which in that reference is said to follow from manipulations of Stirling numbers. This equation (3) can also be obtained as a direct consequence of a bivariate form of the exponential formula for exponential generating functions [9, §5.1], using the observation that

$$
\left(e^{x}-1\right)\left(e^{y}-1\right)=\sum_{p, q \geq 1} \frac{x^{p} y^{q}}{p ! q !}
$$

is the exponential generating function for complete bipartite graphs with $p, q \geq 1$, and these are the possible connected components of admissible graphs.

We now review some standard facts on binomial and toric ideals [2]. Let $I$ be a binomial ideal in $\mathbb{C}\left[x_{1}, \ldots, x_{n}\right]$, generated by binomials of the form $x^{v}-x^{w}$ with $v, w \in \mathbb{N}^{n}$. There is a lattice $L_{I} \subseteq Z^{n}$ such that the localization $I_{x_{1} \cdots x_{n}} \subseteq$ $\mathbb{C}\left[x_{1}^{ \pm 1}, \ldots, x_{n}^{ \pm 1}\right]$ has the form $\left(x^{v}-1: v \in L_{I}\right)$, provided that this localization is a proper ideal, i.e. $I$ contains no monomial. If $\phi_{I}: \mathbb{Z}^{n} \rightarrow \mathbb{Z}^{m}$ is a $\mathbb{Z}$-linear map whose kernel contains $L_{I}$, then $\phi_{I}$ provides a multigrading with respect to which $I$ is homogeneous. (If $\operatorname{ker} \phi_{I}=L_{I}$ exactly then $\phi_{I}$ is said to compute the minimal sufficient statistics for the statistical model associated to $I$.)

The condition that a multivariate Laurent polynomial $f \in \mathbb{C}\left[x_{1}^{ \pm 1}, \ldots, x_{n}^{ \pm 1}\right]$ lies in $I_{x_{1} \cdots x_{n}}$ can be expressed in terms of a graph $\Gamma^{\prime}$, whose vertices are $\mathbb{Z}^{n}$ and whose edge set is $\left\{(v, w): x^{v}-x^{w}\right.$ is a Laurent monomial multiple of a generator of $\left.I\right\}$; in the statistical context these edges are known as moves. To wit, $f$ is in $I_{x_{1} \cdots x_{n}}$ if and only if, for each connected component $C$ of $\Gamma^{\prime}$, the sum of the coefficients on all monomials $x^{v}$ with $v \in C$ is zero. In particular $I_{x_{1} \cdots x_{n}}$ is determined by the partition of $\mathbb{Z}^{n}$ into connected components of $\Gamma^{\prime}$. Note that this partition refines the partition of $\mathbb{Z}^{n}$ into fibers of $\phi_{I}$, for any map $\phi_{I}$ as in the last paragraph. If we are concerned with membership in $I$ rather than $I_{x_{1} \cdots x_{n}}$, analogues of everything in this paragraph are true if we substitute $\mathbb{N}^{n}$ for $\mathbb{Z}^{n}$ and use ordinary monomials rather than Laurent monomials in defining the edges. We will denote the resulting graph on $\mathbb{N}^{n}$ by $\Gamma(I)$, and its induced subgraph on a subset $F \subseteq \mathbb{N}^{n}$ by $\Gamma_{F}(I)$.

Any prime binomial ideal $I$ is equal to the toric ideal $I_{A}$ of a lattice point configuration $A$, where $I_{A}$ is the kernel of the monomial map whose monomials are the points of $A$. Sturmfels shows in [8] that the radicals of the monomial initial ideals of $I_{A}$ are exactly the Stanley-Reisner ideals of regular triangulations of $A$. The StanleyReisner ideal $I_{\Delta}$ of a simplicial complex $\Delta$ on a vertex set $T$ is the monomial ideal of $\mathbb{C}\left[x_{t}: t \in T\right]$ generated by the products of variables $x_{t_{1}} \cdots x_{t_{k}}$ for which $\left\{t_{1}, \ldots, t_{k}\right\}$ is not a face of $\Delta$. Primary decompositions of Stanley-Reisner ideals are easily described: $I_{\Delta}$ is the intersection of the ideals $\left(x_{t}: t \notin F\right)$ over all facets $F$ of $\Delta$.

Sturmfels treats explicitly the $2 \times 2$ determinantal ideal of an $r \times s$ matrix, which is the toric ideal $I_{A}$ for $A$ the set of vertices of the product $\Delta_{r-1} \times \Delta_{S-1}$ of two simplices. 
Theorem 4 (Sturmfels [8]) Let I be the ideal of $2 \times 2$ minors of an $r \times s$ matrix of indeterminates $Y=\left(y_{i j}\right)$. For any term order $\prec$, in $_{\prec} I$ is a squarefree monomial ideal.

Remark 5 If $\prec$ is the revlex term order on the $y_{i j}$, set up analogously to $\prec_{\mathrm{dp}}$, then $\Delta$ has a pleasant description [8]: it is the staircase triangulation. The facets of $\Delta$ are the sets $\pi$ of entries of the matrix $Y$ which form maximal ("staircase") paths through $Y$ starting at the upper left corner, taking steps right and down, and terminating at the lower right corner. Note that staircase paths are maximal subsets of indeterminates not including both $y_{i j^{\prime}}$ and $y_{i^{\prime} j}$ for any $i<i^{\prime}$ and $j<j^{\prime}$. Thus the associated primes of in ${ }_{\prec} I$ are generated by minimal sets of variables $y_{i j}$ which include at least one of $y_{i j^{\prime}}$ and $y_{i^{\prime} j}$ whenever $i<i^{\prime}$ and $j<j^{\prime}$.

The significance of the ideals $P_{C}^{(1)}$ of connected components comes from the following fact.

Fact 6 If $G$ is an admissible graph, then (1) expresses $P_{G}$ as a sum of primes in disjoint sets of variables.

Indeed, $P_{G}^{(0)}$ is the irrelevant ideal in the $p_{i j k}$ with $(j, k) \notin \operatorname{Edges}(G)$, and for each connected component $C$ of $G$ with $s$ left and $t$ right vertices, $P_{C}^{(1)}$ is the $2 \times 2$ determinantal ideal of the $r_{1} \times s t$ matrix of indeterminates $\left(p_{i j k}\right)$ where the row indices are $i \in\left[r_{1}\right]$ and the column indices $(j, k) \in \operatorname{Edges} C$. The irrelevant ideal can mostly be ignored, and so this fact reduces many of our considerations to handling $2 \times 2$ determinantal ideals. (Note that the hypothesis that $G$ be admissible is needed, since otherwise $P_{G}^{(1)}$ includes variables corresponding to nonedges of $G$. We could amend the definition of $P_{G}$ to salvage Fact 6 , but we would lose the also important fact that the summands are determinantal.)

For a first immediate application, by Theorem 4 the in $\prec_{\mathrm{dp}} P_{G}$ are squarefree monomial ideals, implying the radicality claim of Theorem 1 .

For a second, we recover the primary decomposition of $\operatorname{in}_{\prec} P_{G}$ for an arbitrary admissible graph $G$. Let the connected components of $G$ be $C_{1}, \ldots, C_{l}$. Fact 6 implies that $\operatorname{in}_{\prec} P_{G}=$ in $_{\prec} P_{G}^{(0)}+\sum_{i}$ in $_{\prec} P_{C_{i}}^{(1)}$. It then also gives us that if in $_{\prec} P_{C_{i}}^{(1)}=$ $\bigcap_{j} Q_{C_{i}, j}$ are primary decompositions of the $\operatorname{in}_{\prec} P_{C_{i}}^{(1)}$, then

$$
\operatorname{in}_{\prec} P_{G}=\bigcap_{\mathbf{j}}\left(P_{G}^{(0)}+\sum_{i=1}^{l} \operatorname{in}_{\prec} Q_{C_{i}, j_{i}}\right)
$$

is a primary decomposition of $P_{G}$, where $\mathbf{j}=\left(j_{1}, \ldots, j_{l}\right)$ ranges over the Cartesian product of the index sets in $\bigcap_{j} Q_{C, j}$.

Lemma 7 Let $G$ and $G^{\prime}$ be subgraphs of $K_{r_{2}, r_{3}}$. Then $P_{G^{\prime}} \subseteq P_{G}$ if and only if $\operatorname{Edges}(G) \subseteq \operatorname{Edges}\left(G^{\prime}\right)$ and every connected component of $G^{\prime}$ is a union of connected components $C_{1}, \ldots, C_{l}$ of $G$ such that at most one $C_{i}$ contains more than one vertex. 
In particular, for any subgraph $G$ of $K_{r_{2} r_{3}}$ there exists an admissible graph $G^{\prime}$ such that $P_{G^{\prime}} \subseteq P_{G}$. Such a $G^{\prime}$ can be constructed from $G$ as follows: add to $G$ one new edge incident to each of its isolated vertices, and then complete each connected component of the new graph to a bipartite complete graph.

Proof First suppose the consequence fails. Then either

(1) $G$ contains an edge not in $G^{\prime}$, or

(2) some connected component of $G$ is not contained in a single connected component of $G^{\prime}$, or

(3) a connected component of $G^{\prime}$ contains two connected components of $G$ both larger than one vertex.

In case (1), let $(j, k)$ be an edge of $G$ not in $G^{\prime}$. Then $p_{1 j k} \in P_{G^{\prime}}$, but $p_{1 j k} \notin P_{G}$, since Remark 3 describes points in $V\left(P_{G}\right)$ with $p_{1 j k} \neq 0$. Case (2) implies case (1). And in case $(3)$, let $(j, k)$ and $\left(j^{\prime}, k^{\prime}\right)$ be edges of $G$ in different connected components there but in the same connected component of $G^{\prime}$. Then $p_{1 j k} p_{2 j^{\prime} k^{\prime}}-p_{1 j^{\prime} k^{\prime}} p_{2 j k}$ is in $P_{G^{\prime}}$ but not $P_{G}$, again using Remark 3 .

Suppose instead the consequence holds. The generators of $P_{G^{\prime}}^{(0)}$ are in $P_{G}$, since nonedges of $G^{\prime}$ are nonedges of $G$. The generators of $P_{G^{\prime}}^{(1)}$ are also in $P_{G}$. For every pair of edges $(j, k),\left(j^{\prime}, k^{\prime}\right)$ in a connected component of $G^{\prime}$, either all their endpoints are in the same component of $G$ or one of their endpoints is isolated: in the former case $p_{i j k} p_{i^{\prime} j^{\prime} k^{\prime}}-p_{i^{\prime} j k p_{i j^{\prime} k^{\prime}}}$ is in $P_{G}^{(1)}$, in the latter case in $P_{G}^{(0)}$.

Proof of Theorem 1 We first check that the right side of (2) is an irredundant primary decomposition. Let $G$ be an admissible graph. Since $P_{G}$ is a sum of primes in disjoint variables by Fact 6 , it is prime. Irredundance of (2) is the assertion that for $G$ and $G^{\prime}$ distinct admissible graphs, $P_{G}$ is not contained in $P_{G^{\prime}}$. This follows directly from the definition of admissibility and Lemma 7.

So we must prove the intersection statement (2). Let $\prec$ be $\prec_{\mathrm{dp}}$, and write $I=I_{\mathcal{M}}$. It is apparent that

$$
I \subseteq P_{G}
$$

for each $G$ (without using admissibility). Indeed, given a generator $f$ of $I$, without loss of generality of the form $f=p_{i j k} p_{i^{\prime} j^{\prime} k}-p_{i j^{\prime} k} p_{i^{\prime} j k}$, either both edges $(j, k)$ and $\left(j^{\prime}, k\right)$ lie in $\operatorname{Edges}(G)$, in which case $f$ is a generator of $P_{G}^{(1)}$, or one of these edges is not in $\operatorname{Edges}(G)$, in which case $f \in P_{G}^{(0)}$. Therefore the containments

$$
\text { in }_{\prec} I \subseteq \text { in }_{\prec} \bigcap_{G} P_{G} \subseteq \bigcap_{G} \operatorname{in}_{\prec} P_{G}
$$

hold, the intersections still being over admissible $G$. It now suffices to show an equality of Hilbert functions

$$
H\left(S / \mathrm{in}_{\prec} I\right)=H\left(S / \bigcap_{G} \operatorname{in}_{\prec} P_{G}\right),
$$

forcing these containments to be equalities. 
The lattice $L_{I}$ associated to our $I$ is generated by all vectors of the form $e_{i j k}+$ $e_{i^{\prime} j^{\prime} k}-e_{i j^{\prime} k}-e_{i^{\prime} j k}$ and $e_{i j k}+e_{i^{\prime} j k^{\prime}}-e_{i j k^{\prime}}-e_{i^{\prime} j k}$. The map $\phi=\phi_{I}: \mathbb{Z}^{r_{1} r_{2} r_{3}} \rightarrow$ $\mathbb{Z}^{r_{1}+r_{2} r_{3}}$ sending $\left(u_{i j k}\right)$ to

$$
\left(\sum_{(j, k)} u_{1 j k}, \ldots, \sum_{(j, k)} u_{r_{1} j k}, \sum_{i} u_{i 11}, \ldots, \sum_{i} u_{i r_{2} r_{3}}\right)
$$

has kernel containing $L_{I}$. Therefore we obtain a $\mathbb{Z}^{r_{1}+r_{2} r_{3}}$-valued multigrading on $S$, $\operatorname{deg}_{\phi} p_{i j k}=\left(e_{i}, e_{j k}\right)$, in which $I$ is homogeneous. The $\operatorname{deg}_{\phi}$ multigrading refines the standard grading. We will prove that (5) holds in this stronger context of $\phi$-graded Hilbert functions.

Let $d \in \mathbb{Z}^{r_{1}+r_{2} r_{3}}$ be the multidegree of some monomial, and write its components as $d_{i}$ for $i \in\left[r_{1}\right]$ and $d_{j k}$ for $(j, k) \in\left[r_{2}\right] \times\left[r_{3}\right]$. Let $G(d)$ be the bipartite graph with vertex set $\left[r_{2}\right] \amalg\left[r_{3}\right]$ and edge set $\left\{(j, k): d_{j k} \neq 0\right\}$. We now prove the following two claims:

Claim $1 I_{d}=\left(P_{G(d)}\right)_{d}$.

Claim $2\left(\bigcap_{G \text { admissible }} \text { in }_{\prec} P_{G}\right)_{d}=\left(\text { in }_{\prec} P_{G(d)}\right)_{d}$.

These claims imply

$$
\begin{aligned}
H\left(\text { in }_{\prec} I\right)(d) & =H(I)(d)=H\left(P_{G(d)}\right)(d)=H\left(\text { in }_{\prec} P_{G(d)}\right)(d) \\
& =H\left(\bigcap_{G} \operatorname{in}_{\prec} P_{G}\right)(d) .
\end{aligned}
$$

Thence we conclude that (5) holds, proving Theorem 1.

Proof of Claim 1 Observe first that no polynomial homogeneous of multidegree $d$ can be divisible by any $p_{i j k}$ with $(j, k) \notin \operatorname{Edges}(G(d))$. Accordingly we have $\left(P_{G(d)}\right)_{d}=$ $\left(P_{G(d)}^{(1)}\right)_{d}$, and we will work with $P_{G(d)}^{(1)}$.

Since $I$ and $P_{G(d)}^{(1)}$ are binomial ideals generated by differences of monomials, it will suffice to show that the two graphs $\Gamma_{F}(I)$ and $\Gamma_{F}\left(P_{G(d)}^{(1)}\right)$ of moves on the fiber $F=\phi^{-1}(d)$ have the same partition into connected components. It is clear that $\Gamma_{F}(I)$ is a subgraph of $\Gamma_{F}\left(P_{G(d)}^{(1)}\right)$, since containment (4) implies $I_{d} \subseteq\left(P_{G(d)}\right)_{d}=\left(P_{G(d)}^{(1)}\right)_{d}$.

So given an edge of $\Gamma_{F}\left(P_{G(d)}^{(1)}\right)$, say with endpoints $u, u^{\prime} \in F$, we must show that this edge is contained in a connected component of $\Gamma_{F}(I)$, i.e. that $p^{u}-p^{u^{\prime}} \in I$. We have $u=u^{\prime}+e_{i_{0} j_{0} k_{0}}+e_{i_{\ell} j_{\ell} k_{\ell}}-e_{i_{0} j_{\ell} k_{\ell}}-e_{i_{\ell} j_{0} k_{0}}$ for some $i_{0}, i_{\ell} \in\left[r_{1}\right]$ and some two edges $\left(j_{0}, k_{0}\right),\left(j_{\ell}, k_{\ell}\right)$ of $G(d)$ in the same component. Let $\left(j_{m}, k_{m}\right)_{m=0, \ldots, \ell}$ be the edges of a path in $G(d)$ between these, so that for each $0 \leq m<\ell$ either $j_{m}=j_{m+1}$ or $k_{m}=k_{m+1}$. Assume the $\left(j_{m}, k_{m}\right)$ are pairwise distinct. For each $1 \leq m \leq \ell-1$, let $i_{m}$ be such that $p_{i_{m} j_{m} k_{m}}$ divides $p^{u}$. Such an $i_{m}$ must exist because $d_{j_{m} k_{m}}$ is positive. Then 


$$
\begin{aligned}
& \left(p_{i_{0} j_{0} k_{0}} p_{i_{\ell} j_{\ell} k_{\ell}}-p_{i_{0} j_{\ell} k_{\ell}} p_{i_{\ell} j_{0} k_{0}}\right) p_{i_{1} j_{1} k_{1}} \cdots p_{i_{\ell-1} j_{\ell-1} k_{\ell-1}} \\
& =\sum_{m=0}^{\ell-1} p_{i_{1} j_{0} k_{0}} \cdots p_{i_{m} j_{m-1} k_{m-1}} g_{i_{m+1} j_{m+1} k_{m+1}}^{i_{0} j_{m} k_{m}} p_{i_{m+2} j_{m+2} k_{m+2}} \cdots p_{i_{\ell} j_{\ell} k_{\ell}} \\
& \quad-\sum_{m=0}^{\ell-2} p_{i_{1} j_{0} k_{0}} \cdots p_{i_{m} j_{m-1} k_{m-1}} g_{i_{m+1} j_{\ell} j_{m+1} k_{m+1} k_{m}} \\
& \quad \times p_{i_{m+2} j_{m+2} k_{m+2}} \cdots p_{i_{\ell-1} j_{\ell-1} k_{\ell-1}} p_{i_{0} j_{\ell} k_{\ell}}
\end{aligned}
$$

is in $I$, where to save space $g_{i j k}^{i^{\prime} j^{\prime} k^{\prime}}$ denotes the generator $p_{i j k} p_{i^{\prime} j^{\prime} k^{\prime}}-p_{i j^{\prime} k^{\prime}} p_{i^{\prime} j k}$ of $I$. The binomial $p^{u}-p^{u^{\prime}}$ is a monomial multiple of this binomial, so $p^{u}-p^{u^{\prime}} \in I$.

Proof of Claim 2 There is an admissible graph $G^{\prime}$ such that $P_{G^{\prime}} \subseteq P_{G(d)}$, by Lemma 7. Then $\operatorname{in}_{\prec} P_{G^{\prime}} \subseteq$ in $_{\prec} P_{G(d)}$, which implies $\bigcap_{G \text { admissible }}$ in $_{\prec} P_{G} \subseteq$ in $_{\prec} P_{G(d)}$, one of the containments of the claim.

For the other containment, suppose $p^{u}$ is a monomial of multidegree $d$ belonging to in $_{\prec} P_{G(d)}$. By Remark 5, $p^{u}$ is divisible by some $p_{i j^{\prime} k^{\prime}} p_{i^{\prime} j k}$ for $i<i^{\prime}$ in $\left[r_{1}\right]$ and $(j, k),\left(j^{\prime}, k^{\prime}\right)$ two edges lying in the same connected component of $G(d)$ with $(j, k)<\left(j^{\prime}, k^{\prime}\right)$ lexicographically. Now let $G$ be any admissible graph. If $G(d)$ is not a subset of $G$, then $p^{u}$ is divisible by some indeterminate $p_{i j^{\prime \prime}} k^{\prime \prime}$ with $\left(j^{\prime \prime}, k^{\prime \prime}\right) \notin E(G)$, so $p^{u} \in \operatorname{in}_{\prec} P_{G}$. Otherwise $G(d) \subseteq G$, in which case the edges $(j, k)$ and $\left(j^{\prime}, k^{\prime}\right)$ lie in the same component of $G$, so $p_{i j^{\prime} k^{\prime}} p_{i^{\prime} j k} \in$ in $_{\prec} P_{G}$, implying $p^{u} \in$ in $_{\prec} P_{G}$. Therefore $\operatorname{in}_{\prec} P_{G(d)} \subseteq \bigcap_{G \text { admissible }}$ in $_{\prec} P_{G}$.

Observe finally that Claim 1 alone would suffice for the radicality in Theorem 1, supposing we already knew the $P_{G}$ to be the associated primes. For radicality it suffices that $I$ contain the intersection of its minimal primes, and this follows using Claim 1 one multidegree at a time, since the multidegree $d$ part of this intersection is contained in $\left(P_{G(d)}\right)_{d}$.

Acknowledgements We thank Thomas Kahle for discussions, and Bernd Sturmfels and a patient referee for careful readings and for several helpful suggestions.

Open Access This article is distributed under the terms of the Creative Commons Attribution Noncommercial License which permits any noncommercial use, distribution, and reproduction in any medium, provided the original author(s) and source are credited.

\section{References}

1. Drton, M., Sturmfels, B., Sullivant, S.: Lectures on Algebraic Statistics. Oberwolfach Seminars, vol. 39. Springer, Berlin (2009)

2. Eisenbud, D., Sturmfels, B.: Binomial ideals. Duke Math. J. 84, 1-45 (1996)

3. Grayson, D.R., Stillman, M.: Macaulay2, a software system for research in algebraic geometry. Available at http://www.math.uiuc.edu/Macaulay2/

4. Greuel, G.-M., Pfister, G., Schönemann, H.: Singular 3.0-a computer algebra system for polynomial computations. In: Kerber, M., Kohlhase, M. (eds.) Symbolic Computation and Automated Reasoning, The Calculemus-2000 Symposium, pp. 227-233 (2001) 
5. Greuel, G.-M., Pfister, G.: primdec. 1ib, a Singular 3.0 library for computing the primary decomposition and radical of ideals (2005)

6. Herzog, J., Hibi, T., Hreinsdóttir, F., Kahle, T., Rauh, J.: Binomial edge ideals and conditional independence statements. arXiv:0909.4717

7. Kahle, T.: Binomials.m2, code for binomial primary decomposition in Macaulay2. http:// personal-homepages.mis.mpg.de/kahle/bpd/index.html

8. Sturmfels, B.: Gröbner bases of toric varieties. Tōhoku Math. J. 43, 249-261 (1991)

9. Stanley, R.P.: Enumerative Combinatorics, vol. 2. Cambridge Studies in Advanced Mathematics, vol. 62. Cambridge University Press, Cambridge (1997)

10. Studený, M.: Probabilistic Conditional Independence Structures, Information Science and Statistics. Springer, New York (2005) 